\title{
Téoros
}

Revue de recherche en tourisme

\section{Le tourisme rural - entre certitudes et improvisations}

\section{Jacques Perret}

Volume 20, numéro 2, été 2001

Tourisme rural

URI : https://id.erudit.org/iderudit/1071638ar

DOI : https://doi.org/10.7202/1071638ar

Aller au sommaire du numéro

Éditeur(s)

Université du Québec à Montréal

ISSN

0712-8657 (imprimé)

1923-2705 (numérique)

Découvrir la revue

Citer cet article

Perret, J. (2001). Le tourisme rural - entre certitudes et improvisations. Téoros, 20(2), 15-21. https://doi.org/10.7202/1071638ar d'utilisation que vous pouvez consulter en ligne.

https://apropos.erudit.org/fr/usagers/politique-dutilisation/ 


\section{Le tourisme rural - entre certitudes et improvisations}

\section{Jacques Perret}

C et article est né d'un constat : chaque fois qu'un développement touristique est projeté, les discours et les méthodes d'action se répètent et le même décalage se produit entre, d'une part, des décideurs (administration et élus) qui proposent un développement et, d'autre part, des professionnels du tourisme (entrepreneurs) qui sont à l'écart des projets bien qu'ils soient directement concernés. Comment interpréter cette situation? Nous verrons, dans un premier temps, la rationalité mise en œuvre par les développeurs et celle des créateurs d'entreprise. Dans un second temps, nous aborderons les conséquences de cette appropriation du développement par des décideurs sûrs de leur vérité.

\section{Des diagnostics identiques}

Les projets de développement touris-tique reposent sur un diagnostic préalable. Les auteurs du diagnostic déclarent leur intention de tenir compte de la situation particulière à la petite région considérée ; pourtant, il faut bien constater que les termes du diagnostic sont toujours les mêmes : le territoire est enclavé, l'agriculture vieillit, les autres activités sont en déclin, les jeunes ne trouvent pas d'emploi, les services sont menacés. En revanche, de nombreuses ressources ne sont pas exploitées : des savoir-faire sont oubliés, les paysages sont magnifiques et il y a des espaces libres. Il apparaît évident que la région possède un potentiel important qui est peu mis en valeur (faible capacité d'hébergement, manque d'activités d'ac- compagnement, absence d'information), qui est mal valorisé (chambres d'hôtels d'un autre âge, fermeture des services et des commerces aux heures de flux touristiques, commercialisation parcellisée, etc.) ou, pire encore, le potentiel touristique se dégrade (fermeture du paysage avec l'avancée de la forêt, constructions neuves qui ne respectent pas le style local, etc. La cause de ces évolutions est toujours la même : les gens du pays ne sont pas conscients de la richesse de leur pays et ils ne savent pas faire du tourisme; c'est un tourisme de cueillette. En conclusion, le diagnostic propose un plan de développement touristique dans lequel il est recommandé d'inventorier le patrimoine, de définir des produits, de mettre au point une information, une promotion et une commercialisation adaptées, de former les acteurs pour qu'ils deviennent de vrais professionnels et qu'ils soient en mesure de sensibiliser tous les acteurs locaux concernés par le projet.

Cette similitude dans les diagnostics soulève la question : comment se fait-il qu'à l'heure où les décideurs, les théoriciens, reconnaissent et prônent la diversité des modes de développement, tous les diagnostics constatent la fragilité de l'économie locale et la nécessité de lancer un projet de développement touristique qui s'avère toujours particulièrement urgent? Ce regard unique n'est pas fortuit. Il est le produit d'un schéma de pensée qui se nourrit de la même inspiration.

\section{La pensée unique}

La définition du projet de développement et celle de son processus d'élaboration reposent sur des postulats. Ils concernent les conditions du diagnostic, la notion de développement, la fonction de l'espace rural, les méthodes employées.

\section{Le développement par la croissance}

Le développement est décliné dans sa version strictement économique. L'accroissement quantitatif des activités est un $a$ priori nécessaire. C'est la croissance qui apporte le bien-être, le progrès. Pour G. Rist, cette notion « s'inscrit dans le prolongement de cette croyance ordinaire qui a inspiré [depuis la dernière guerre] l'extension de la société marchande, y compris dans son expression coloniale» (1996 : 348). Le projet de développement consiste toujours à augmenter la fréquentation touristique, en agissant sur la capacité d'accueil, les activités récréatives, l'étalement des saisons. Toute solution est bonne à partir du moment où elle permet de retenir les touristes et d'en attirer de nouveaux. Les services permanents, les transports, sont conçus pour favoriser l'accessibilité, attirer de nouveaux entrepreneurs, créer des « avantages comparatifs ».

Dans cette version économique, l'espace se découvre une vocation récréative. La fonction agricole est satisfaite, la fonction industrielle et artisanale apparaît illusoire avec la forte concentration des activités, sauf pour les cas particuliers de forte tradition industrielle. Reste la fonction de services : environnement, santé et tourisme. Cette vocation suppose la création de ressources : hier les thermes, les domaines skiables, les plages, les plans d'eau, 
aujourd'hui les paysages et le patrimoine vernaculaire. On s'intéresse à l'environnement grâce à sa valeur marchande.

Ce développement dépend du marché et, naturellement, de la demande : « [...] on part toujours de la demande, des clients, des marchés et des études avant de concevoir les produits, les prix, la commercialisation [...] » (Peter Drucker, cité in AFIT, 2000 : 97).

On postule que la demande dispose toujours d'un fort potentiel de croissance. Par exemple, un rapport au Ministre du Tourisme de 1993, constatant que la fréquentation touristique ne concerne que $20 \%$ du territoire et que seulement $22 \%$ des dépenses touristiques profitent à l'espace rural, conclut : «Il y a dans l'espace français un potentiel de développement touristique utile au plan économique et de l'emploi mais aussi intéressant pour rééquilibrer la vie sociale» (Descamps, 1993 : 4). Ces prévisions résultent le plus souvent de la prolongation des tendances passées, parce qu'on n'a pas les moyens ni le temps de faire une étude de marché et parce qu'on croit aux tendances lourdes. Le même rapport reconnaît qu'il est « difficile de faire en matière touristique des prévisions chiffrées d'évolution de la demande et d'en tirer des conclusions sur les dépenses qui l'induiront [...]»; il retient cependant que l'augmentation relativement modeste de la fréquentation des cinq dernières années semble «la tendance naturelle que l'on constate actuellement » et, en conséquence, on peut compter sur une augmentation probable de 44 à 64 millions de nuitées, induisant une hausse des dépenses de 7,4 à 10,6 milliards de francs, entraînant la création nette de 10000 à 15000 emplois, soit une croissance de $10 \%$ en 5 ans. Le rapport conclut que si un effort volontariste est réalisé (dont le rapport présente les modalités nécessaires), «[...] c'est probablement 50000 emplois nouveaux qui seraient créés, et s'ajouteraient aux emplois difficilement chiffrables qui, sans cela, disparaîtraient » (Descamps, $1993: 5$ ). Ce type de prévisions peut se vérifier, mais dans le cas présent, le taux de départ des Français en séjours est resté stable dans les années 1990 et le nombre de nuitées a même diminué de $5 \%$ de 1994 à 1998) (AFIT, 2000). Malgré tout, la croissance de l'offre s'est poursuivie.

\section{Une méthodologie rationnelle à l'échelle territoriale}

La mise en œuvre du développement rural tel qu'il est défini doit répondre, pour ses promoteurs, à un certain nombre de conditions, sous peine de mettre en péril la réussite du projet. Le développement est l'affaire d'un pays, d'une société locale, d'un territoire. Il est élaboré dans un périmètre délimitant une entité qui s'impose par ses liens économiques, son histoire, son organisation politique (Marcelpoil, 1997). Le développement ne peut avoir lieu que si un tel ensemble existe, s'il a une identité, une cohésion sociale. En cas de défaillance, le groupe doit être structuré, animé ; le lien social est construit par l'animation ; l'identité est affirmée par des événements symboliques, l'image, le logo. Le besoin de cohérence requiert une direction commune pour décider, coordonner, faire appliquer ; c'est le rôle donné à la structure de développement qui crée pour l'occasion un groupe de pilotage du projet et des groupes de réflexion thématique. Le territoire est ainsi considéré comme une entreprise (AEIDL, 1997), par son objectif économique et son mode d'organisation, dans laquelle l'ensemble des acteurs dans un même projet doit se fondre.

Le projet est global ; il concerne tous les aspects touchés de près ou de loin par le tourisme. On postule que la bonne santé de l'ensemble assure le bien-être des parties. On mesure la situation et la dynamique globales. L'évaluation est réalisée par des moyennes. Le projet est cohérent ; un seul projet (le projet touristique) concourt à un but commun. Les diverses actions du projet sont hiérarchisées, calées dans le temps, coordonnées dans un programme d'ensemble. Les initiatives en cours dans le territoire sont soupçonnées d'individualisme et d'incohérence ; elles sont négligées ou canalisées dans le projet.

Dans de telles conditions, le développement ne peut être spontané. L'acteur éco- nomique de base, sur le terrain, n'a pas la connaissance; il ne sait pas faire. Il n'a pas conscience de ce qu'il pourrait faire, de ce qu'il doit faire. Il est englué dans ses traditions, ses habitudes et ses croyances. Il doit être informé, sensibilisé, formé. Il doit se laisser guider par ceux qui savent. Tant qu'il n'a pas la compétence nécessaire, il doit recourir à des spécialistes, à des techniciens, à des consultants et à d'autres chercheurs.

Alors comment se fait-il que ce schéma de développement, si bien construit, ne séduise pas toute une foule d'entrepreneurs ? Pour le comprendre, nous avons fait l'analyse d'une longue série d'entretiens, réalisés au cours des dernières années auprès d'acteurs du tourisme, essentiellement des entrepreneurs privés, mais aussi des particuliers regroupés au sein d'une association. Ces acteurs sont très diversifiés et leur démarche évolue au cours de leur histoire ; pour simplifier, nous avons retenu la période de création et de montée en puissance de leur activité. C'est une période qui nous est apparue particulièrement significative puisqu'elle correspond à une démarche de création, celle que cherchent à initier les plans de développement touristique. L'histoire de ces créateurs fera percevoir que la logique de développement de leur activité ne répond pas aux conditions souhaitées par le schéma des développeurs présenté ci-dessus.

\section{D'autres rationalités}

\section{Des motivations complexes}

Qui sont ces entrepreneurs? C'est, par exemple, un cuisinier et sa femme serveuse qui ne supportent plus les conditions de travail dans un grand restaurant en ville : ils achètent un hôtel-restaurant en pleine campagne, pour le cadre de vie, le calme, pour vivre avec leurs enfants. C'est une femme d'exploitant agricole qui fait de l'accueil à la ferme : une chambre d'hôtes, un camping ou un atelier de transformation de palmipèdes gras ; ayant hésité à reprendre son activité salariée alors que ses enfants sont encore en bas âge, elle a décidé de monter son propre atelier, pour rester chez elle tout en étant indépendante 
de son conjoint. C'est un groupe de jeunes amateurs de spéléologie qui achètent et aménagent une grotte, pour le plaisir de s'adonner à leur passion en toute liberté, de faire partager leur plaisir et la beauté de la grotte. Ce sont les jeunes d'un village qui créent une petite station de sports d'hiver ou un centre-école de ski de fond pour éviter de partir ; ils veulent montrer qu'ils ne sont pas de pauvres gueux et qu'ils peuvent eux aussi se moderniser.

Quels sont les points communs de ces initiatives apparemment très disparates? Un premier constat s'impose. Tous ces créateurs d'entreprises ont d'abord voulu satisfaire un désir : une volonté d'indépendance, un besoin d'exister, le plaisir de faire, le choix de rester au pays. La motivation n'est pas strictement économique ; c'est davantage une question de dignité, de liberté. Le revenu attendu est un moyen de satisfaire ce désir premier. C'est cette volonté fondamentale qui va guider, au moins dans les premières années, le mode de développement de l'entreprise.

Deuxième constat, tous raisonnent à leur échelle, celle de leur entreprise, dans le cadre familial ou amical. Ils réalisent quelque chose pour satisfaire leurs besoins personnels. Ils n'ont pas l'ambition de faire du développement, à l'échelle de leur commune ou de leur pays, encore moins de leur territoire, ce « concept d'intellectuel». Ils tiennent compte de leur environnement (la fréquentation touristique, la demande visible, les activités existantes) pour trouver leur place, mais ils ne l'intègrent pas comme condition de leur réussite ; cela viendra après, quand leur affaire sera bien installée, quand l'évolution de leur entreprise dépendra de l'orientation touristique du pays.

\section{L'improvisation comme méthode}

Ces entrepreneurs n'ont pas de projet, à proprement parler, fait de budgets prévisionnels, d'objectifs économiques, de plans, d'échéances, ni de stratégie formulée dans un programme cohérent et global. Ils improvisent. Leur méthode est d'être à l'écoute permanente de la situation, des opportunités comme des problèmes. À force d'information, d'exemples, de coups de cœur, d'émotions, de rencontres, d'opportunités, en fonction des contraintes qui se présentent, ils décident. Ils ne sont pas sûrs de leur choix ; ils savent qu'ils peuvent se tromper. Ils sont en alerte continue, d'autant plus qu'ils se savent regardés, par les voisins, les sérieux, ceux qui sont du métier. Même après coup, une fois installés, quand ils regardent derrière eux, ils ne peuvent pas vraiment expliquer leur chemin... succession d'idées, de situations, d'opportunités, de problèmes. La raison profonde est sans doute dans la volonté de se réaliser, de se prouver quelque chose, tout en se distinguant des autres.

Ils raisonnent à court terme, voire dans l'urgence : «mon projet est à huit jours ». Ils ont des idées pour la suite, multiples, contradictoires ; ils attendent pour décider : «on verra bien ». Ils ont bien sûr l'intention de durer, mais ce ne serait pas une catastrophe si l'activité s'arrêtait demain. Ils auront eu la satisfaction d'avoir tenté, d'avoir eu le courage de le faire. La réussite à long terme, ils n'y pensent pas ou ils en rêvent; ils n'ont pas de programme précis pour y parvenir.

\section{Maîtriser}

Ces créateurs font par eux-mêmes. C'est une façon de dépenser moins, de limiter les investissements, de compenser une absence de moyens financiers, mais c'est aussi une façon de réaliser quelque chose. L'important est que ce sont eux qui décident et ce sont eux qui s'engagent. Ils veulent mâtriser leur affaire ; ils ne s'en remettent pas à des techniciens, ils ne commandent pas d'études préalables. Ils glanent des renseignements un peu partout, font leur propre synthèse et choisissent intuitivement la solution qui parait la plus adaptée à leur situation et à ce qu'ils veulent faire. C'est une conviction : ils sentent que ça va marcher. C'est un pari : leur budget prévisionnel est sommaire. Après coup, ils pensent qu'il fallait être fou pour se lancer, mais ils ne regrettent rien ; cela ne pouvait pas se faire autrement.

\section{Progressivement}

Faire par eux-mêmes c'est aussi réaliser petit à petit. Le manque de moyens oblige à investir progressivement, au fur et à mesure des entrées d'argent. Cette faiblesse peut devenir un avantage ; ils suivent le marché de très près, ils s'adaptent d'une année à l'autre, ils expérimentent diverses formules, ils peuvent revenir en arrière parce que l'investissement est modeste et réutilisable autrement. Ce mode de production, aux investissements modestes et étalés dans le temps, a aussi l'avantage, par la force des choses sinon par conviction, de provoquer moins de conséquences sur l'environnement.

\section{Hors normes}

Leur façon de faire peut être un défi dans la mesure où ils prennent à contre-pied les normes en vigueur et les avertissements des conseilleurs. L'entreprise devient un défi quand ils se lancent malgré les avis contraires, sans les subventions éventuelles ni le soutien des banques. Ils sont obligés de réussir pour montrer qu'ils « avaient raison ».

L'économie est un moyen ; elle est aussi une sanction. Il faut que l'entreprise roule, qu'elle fasse des bénéfices, qu'elle investisse, qu'elle se modernise. Un arrêt serait une déception, la sanction d'une folie, la preuve que ceux qui ne les ont pas aidés avaient raison. Réussir dans le métier, c'est aussi réussir par rapport aux normes; être simplement satisfait n'apporte pas la reconnaissance sociale (Dimou, 1989).

Ces entrepreneurs répètent qu'ils ont agi seuls. Évidemment, ils n'ont jamais été vraiment seuls comme ils le disent spontanément, parce qu'ils ont bénéficié de soutien, d'information, de coups de main, de prêts, et ce de la part de leur conjoint, de leur famille, de leurs voisins ou de leurs connaissances. Ils ont bénéficié de la confiance de leurs proches ou d'autres qui leur ressemblent, qui partagent une même situation ou les mêmes valeurs ; par exemple, les migrants (ceux qui sont originaires d'une autre région) forment un réseau informel d'entraide. Ils disent qu'ils ont été seuls parce qu'ils n'ont pas été aidés par aucun service public. Ils n'ont pas reçu d'aide publique parce qu'elle n'existait pas à leur époque, parce qu'ils ne rencontraient 
pas les conditions requises pour l'obtenir ou parce qu'ils ne l'ont pas cherchée ou trouvée. Ils ont subi les conséquences d'être hors normes, mais, d'un autre côté, ils sont fiers d'avoir inventé quelque chose et de l'avoir fait, en dépit de tout.

\section{Des rationalités opposées}

Les logiques professionnelles de ces créateurs d'entreprise se révèlent ainsi fort éloignées de la démarche programmée par les projets de développement. Tout les oppose : le plaisir de faire par opposition à la croissance, l'opportunisme face au plan, le doute face aux vérités, l'improvisation face aux certitudes.

L'une et l'autre de ces logiques apparaissent rationnelles; il n'y a pas d'un côté un schéma de développement raisonné, cohérent, global et, de l'autre, des logiques considérées, avec un brin de sentiment de supériorité, comme irrationnelles, voire archaïques.

Cette opposition est fréquente dans les projets de développement local, mais estce toujours le cas avec le développement durable qui renouvelle les concepts et les priorités du développement?

\section{Un ordre nouveau : le tourisme durable}

Il existe diverses versions officielles du tourisme durable. Nous retiendrons ici la définition et la méthodologie élaborées et prônées par la Fédération des Parcs $\mathrm{Na}$ turels Régionaux (PNR) qui veut tenir un rôle d'avant-garde en la matière grâce à ses expérimentations dans ses territoires, en France comme en Europe, avec la Charte européenne du tourisme durable dans les espaces protégés (1997).

Cette charte, fondée notamment sur la Charte mondiale du tourisme durable signée à Lanzarote en 1995, a pour objectif de développer un tourisme de qualité, durable, défini comme

[...] toute forme de développement, aménagement ou activité touristique qui respecte et préserve à long terme les ressources naturelles, culturelles et sociales, et contribue de manière positive et équitable au développement économique et à l'épanouissement des individus qui vivent, travaillent ou séjournent dans les espaces protégés.

\section{Un développement global}

Le tourisme durable, c'est d'abord et toujours du développement économique. C'est par cette sacro-sainte dynamique de la création d'activités productives que l'on attend le progrès, l'épanouissement de l'homme. En conséquence, le patrimoine naturel et culturel des parcs et des autres espaces protégés ne peut pas être considéré autrement que comme une ressource, un capital à fort potentiel touristique (principe 1 de la charte). L'existence d'un marché est même considérée comme un mode de valorisation des ressources naturelles ; c'est peut-être même une chance ( $c f$. la sauvegarde de la faune africaine dans des parcs justifiés et alimentés par le tourisme et, plus près de nous, la nouvelle mode des produits du terroir annoncés «biologiques »). Le tourisme durable est un nouveau fonds de commerce, un nouveau créneau, avec ses produits, ses circuits de commercialisation, sa publicité...

Cependant, le tourisme durable innove parce qu'il recommande des pratiques nouvelles, en termes environnemental et social. Il suppose bien sûr la préservation du patrimoine naturel (principes 2 et 3 ). Il reprend le postulat que le tourisme « contribue de manière positive au développement économique local », mais pas à n'importe quelles conditions : il s'oppose implicitement aux implantations ex nihilo et recommande une intégration dans l'économie locale (principe 4); il prône un équilibre entre tourisme et autres activités, dites traditionnelles. Le développement est ici envisagé d'une manière différente, peu intensive, en relation avec les autres activités, dans le respect des touristes et des accueillants. Il reconnaît de nouvelles formes d'emploi, est attentif aux conditions de travail des salariés et à l'accueil des clientèles.
Le tourisme durable apparaît comme une variété de développement local, respectueux de l'environnement, des hommes, mais c'est toujours du développement économique. Face aux abus et à la perversité du tourisme de masse, les promoteurs du tourisme durable veulent moraliser une activité commerciale ou, mieux, ils ont l'intention, tout au moins dans certaines versions, de redonner du sens à l'activité économique, de remettre l'économie au service de l'homme (Teyssandier, 2000).

Cette proposition de développement n'est pas simple à mettre en œuvre. Ses promoteurs proposent une stratégie et des méthodes adaptées : «[...] il s'agit d'une approche globale [...] qui doit donc se fonder sur un diagnostic complet des besoins d'un territoire et de ses potentiels touristiques »; «[...] il faut planifier, gérer et évaluer régulièrement» (principe 1).

\section{Méthode identique}

La méthode reprend celle du développement local : le projet de tourisme durable est élaboré à l'échelle territoriale et selon une stratégie qui comprend plusieurs phases bien définies :

1. identifier les grands enjeux du développement touristique durable,

2. élaborer le diagnostic du territoire,

3. évaluer les coûts de réalisation de la stratégie et les moyens disponibles,

4. reformuler les objectifs et opérer les choix stratégiques en fonction des moyens disponibles et des priorités,

5. définir les indicateurs et les outils de suivi et d'évaluation de la stratégie,

6. définir le programme d'actions (Fédération des PNR, 2000).

Le diagnostic lui-même comprend l'inventaire du patrimoine local, le diagnostic économique et social, touristique, le diagnostic des enjeux d'amélioration du cadre de vie et de maintien des services, l'étude des flux de visiteurs, le diagnostic de l'éducation à l'environnement. Le programme d'action comprend : l'amélioration de la qualité de l'offre, la création d'une offre 
touristique spécifique, la sensibilisation du public, la formation, la préservation et l'amélioration du cadre de vie et le maintien des services, la protection et la mise en valeur du patrimoine, le développement économique et social, la maîtrise de la fréquentation touristique, le suivi et l'évaluation du programme d'actions.

Le bien fondé de cette stratégie n'est pas en cause, mais cet énoncé des chapitres permet simplement de constater que les modalités de mise en œuvre du tourisme durable sont à cent lieues des préoccupations des créateurs d'une entreprise. C'est une globalité à une autre échelle, le territoire, qui veut tout prévoir, pour un temps déterminé, de façon à programmer les procédures, les financements, les réunions de travail, etc. Rien n'est laissé au hasard, aux changements de perception. Les choix sont faits pour la durée du plan, cinq ans en général.

Développement local ou développement durable, la procédure canonique du développement est la même ; elle repose sur un projet économique, planifié, qui s'oppose à la démarche tâtonnante du créateur, faite d'improvisations, de sensations, de plaisir de créer. Concrètement, sur le terrain, ces démarches se côtoient, s'ignorent, se servent l'une de l'autre. Mais une seule est reconnue, celle portée par les services spécialisés qui s'accaparent la bonne pratique du développement.

\section{L'accaparement}

Les créateurs d'entreprise n'ont pas conscience de faire du développement, donc ils n'en parlent pas. Le développement, que l'on peut définir comme toute forme de changement social contribuant à l'amélioration des conditions de vie, n'est pas identifié en tant que tel ; il est réduit aux politiques publiques, aux services de développement, à ceux qui sont chargés de lancer des opérations de développement, de gérer des fonds de développement, nationaux ou européens, d'organiser la concertation avec l'ensemble des acteurs concernés pour le développement de tel secteur d'activité ou de tel territoire. Le développement se confond ou se limite à ce type d'interventions. Certains pensent que le développement local a été créé dans les années cinquante, quand l'État a commencé à intervenir pour financer des opérations économiques localisées. Il est donc admis que le développement local est l'affaire des acteurs publics, techniciens ou élus ; il appartient à ceux qui en parlent, à ceux qui affirment en faire. Ceux-ci ont la légitimité pour représenter l'intérêt général ; ils ont les moyens d'agir : ils ont le temps, l'expérience, les relations, la connaissance des circuits financiers et administratifs. Ils peuvent donc imposer leur propre vision du développement, leurs choix d'opérations prioritaires. Et ce, d'autant plus que les procédures deviennent de plus en plus lourdes, en termes de programmation, de suivi et de contrôle, pour justifier des spécialistes du développement ; les agents de développement qui pensaient être des animateurs sont réduits à être des secrétaires du développement, confinés dans leur bureau, au lieu de rencontrer les professionnels et de répondre à leurs attentes.

Cet accaparement du développement par des organismes spécialisés a trois conséquences qui s'entretiennent mutuellement : la bonne solution, la bureaucratie et le marketing en guise de professionnalisation.

\section{La bonne solution}

Les développeurs, qu'ils soient élus, techniciens ou théoriciens, sont persuadés de détenir la vérité ou la meilleure solution. L'adhésion au schéma dominant est rassurante. Pour inciter au tourisme durable, les développeurs définissent « la meilleure approche $»$ qui va permettre un équilibre entre des objectifs de protection et de développement social, culturel et économique. On est sûr de son idée, d'autant plus qu'elle est généreuse. On ne s'intéresse pas aux autres représentations du développement ; au contraire, tout ce qui n'apparaît pas comme un comportement rationnel est considéré comme un obstacle, quelque chose d'irrationnel.

Les décideurs ont l'ambition, généralement de bonne foi, de mettre en place une dé- marche objective. Les méthodes sont travaillées, cent fois remises sur le tapis de façon à être très performantes, pour s'adapter aux mentalités des acteurs. Elles consistent en fait à affiner la méthode qui va permettre au service de développement de mettre en pratique sa propre idée du développement (Marcelpoil et Perret, 1999). Celle-ci est tellement évidente qu'elle n'est pas définie en préalable des diagnostics, dans quelques pages de méthodologie. Le développement local est considéré comme une pratique, dénuée de toute idéologie (Pecqueur, 1989), alors qu'il est pourtant lourdement chargé de sens.

Corrélativement, les socioprofessionnels sont perçus comme des acteurs privés raisonnant à l'échelle de leur entreprise, à court terme, de façon individuelle, donc indisponibles, voire incapables, sauf exception, de concevoir et de porter une action de développement. Ils sont considérés inaptes, au point que des structures de développement se considèrent comme la matière grise du développement. Ils n'ont pas le temps de participer aux politiques de développement. C'est vrai qu'ils n'ont souvent pas le temps de consacrer des journées de réflexion à des démarches collectives ; il est vrai aussi, sur un plan plus fondamental, qu'ils raisonnent autrement, qu'ils ne sont pas compétents pour traiter de problèmes qui ne sont pas les leurs et qu'ils ne connaissent rien aux procédures administratives. Ils se tiennent à l'écart ; ils se sentent mal représentés ou ne se sentent pas concernés par les divers services existants et ils en attendent peu de choses ; de leur côté, les techniciens expriment leurs difficultés à travailler avec les professionnels, souvent inorganisés, raisonnant à l'échelle de leur entreprise et soucieux de la vie de leur entreprise, voire de sa survie.

Cette ambition des services publics n'a pourtant pas de fondement historique. Les organismes de développement sont récents (quelques décennies à peine), et les premières formes de développement touristique sont beaucoup plus anciennes. Les grands pôles touristiques traditionnels, que ce soit Chamonix, Val d'Isère, la Côte d'Azur, sont nés d'un foisonnement d'ini- 
tiatives privées, souvent hors normes, hors de tout cadre administratif de développement qui n'existait pas à l'époque. C'est leur succès et leurs excès qui imposent une régulation. À l'inverse, les grandes opérations de développement touristique soutenues par l'État ne sont pas forcément des réussites exemplaires : les services publics eux-mêmes s'interrogent pour trouver un avenir aux stations de montagne ou du littoral créées de toutes pièces dans les années 1970. Les réussites et les erreurs ne sont donc pas liées au statut de leurs auteurs. De leur côté, les parcs constatent la richesse de leur patrimoine, qu'il s'agisse des paysages, des savoir-faire ou de l'habitat ; ce patrimoine a été construit par les habitants peu à peu. Et ce ne sont pas les abus récents, les dégradations ou les friches, qui justifient de déclarer les professionnels incompétents et, en conséquence, de s'approprier les bonnes pratiques du tourisme durable. Celui-ci est déjà pratiqué depuis belle lurette, et une volonté de diffusion pourrait utilement s'appuyer sur des exemples vivants.

\section{La bureaucratie}

Quant à la nature des procédures de financement ; même si l'on pense que les procédures ne sont pas bonnes, si l'on veut obtenir du financement et, pour cela émarger à tel ou tel programme, solliciter telle subvention, « il faut faire comme ça ».

Le diagnostic réalisé dans le cadre d'un programme de développement est ici évocateur. L'objectif du diagnostic est de montrer que, quelles que soient la région et la période, un développement s'impose. On met donc en avant les handicaps du territoire, ses faiblesses, ses atouts... et le bilan est toujours négatif. Aucun ne s'étale sur la vitalité des initiatives locales ou la richesse du pays. On peut signaler la pauvreté des communes, mais on n'a pas d'information sur les comptes en banque privés. Aucun bilan n'est fait sur la volonté et le besoin de changer. Il est évident qu'il faut changer. Il faut développer. Face aux difficultés de mise en œuvre du projet de développement, on va considérer que les méthodes ou les propositions n'étaient pas bonnes, qu'elles sont arrivées trop tôt, que les acteurs n'étaient pas mûrs. On va donc refaire un projet de développement. Pourtant un autre bilan est possible : en fait, tout va bien comme ça; ils n'ont pas faim. «L'échec ne sanctionne pas les institutions, au contraire on demande des moyens supplémentaires, on élargit, on renouvelle les méthodes, preuve de vitalité qui renforce la légitimité » (Perrot, 1986 : 98).

Le développement s'impose, par son concept, mais aussi par ses services spécialisés. Plus il existe de développeurs, de financements, plus les procédures administratives se multiplient. C'est une raison d'exister pour ceux qui sont chargés du développement. Et cette institutionnalisation du développement tend à les éloigner des préoccupations des professionnels. Les développeurs vont tenter par divers moyens de les convaincre de se développer. Le maître mot en la matière est la professionnalisation.

\section{La professionnalisation ou le marketing indispensable}

Puisque les professionnels ne perçoivent pas la nécessité de se développer, les développeurs tentent de les faire adhérer à leur projet : sensibilisation, formation, toute forme de communication pour leur faire prendre conscience. Conscience qu'ils disposent d'un potentiel touristique, que la qualité des produits n'est pas adaptée à la demande, que la méthode de commercialisation n'est pas efficace. En un mot, il faut passer d'un tourisme de cueillette à un tourisme professionnel. Ici le maître mot est le marketing.

L'objectif premier du marketing est d'accroître son chiffre d'affaires, en tentant de répondre aux besoins de la clientèle. Toutes les techniques sont bonnes pour identifier des niches de clientèles, adopter une tarification attractive, fidéliser la clientèle, aller chercher les clients des concurrents. Il est fortement conseillé de recourir à un expert pour faire un diagnostic et définir la «stratégie d'attaque » (AFIT, 2000).

Pourquoi une telle agressivité ? Ce n'est pas l'évolution de la demande qui la justifie ; le marketing est indispensable pour trouver sa place sur un marché qui est devenu fortement concurrentiel. La demande n' augmente pas; les populations qui ne partent pas en vacances sont toujours aussi nombreuses : $31 \%$ en 1996 (Viard, 1998). Les touristes recourent au maximum à des formules non marchandes. Par contre, l'offre commerciale se multiplie et elle se situe sensiblement dans la même gamme de produits : on vend partout la nature, le calme, le ressourcement, pour attirer une clientèle qui attend de la campagne des contacts (accueil, convivialité), les vraies valeurs (simplicité, authenticité), l'antithèse de la ville (calme, nature, animaux). Le touriste veut à la fois retrouver ses racines (retour aux sources), découvrir un autre monde, une autre vie, des relations humaines et non mercantiles (Gramond et coll., 1998). Les produits s'adressent à la même clientèle, aisée, cultivée, amateur de patrimoine et de racines. Même si aucune statistique n'est publiée, on peut craindre que le tourisme rural n' aboutisse à la même situation que celle des sports d'hiver pour lesquels l'offre est surabondante : en quelques semaines la demande est satisfaite (AFIT, 2000).

Cette perception de la campagne est celle des cadres, intellectuels, cultivés, aisés, amateurs de culture et de loisirs, en famille ou à la retraite. Mais elle ne concerne pas les jeunes qui cherchent d'autres animations ou les familles modestes que les produits haut de gamme repoussent. On formate le tourisme rural selon une clientèle haut de gamme. On ne s'intéresse pas aux familles modestes qui ne partent souvent pas en vacances (Gramond et coll., 1998).

Le marketing est une arme de guerre, entre pays ou entre stations, de plus en plus nombreux sur le marché, qui ont développé des programmes d'équipement et d'hébergement en croyant à l'augmentation continue de la demande. Il est d'autant plus indispensable que la communication doit permettre de présenter une offre originale, dans un pays authentique, alors que l'offre est de plus en plus banale, avec des prestataires dont on a tendance à normaliser les comportements pour plaire à la clientèle $«[\ldots]$ ce qui est décisif, c'est ce que le 


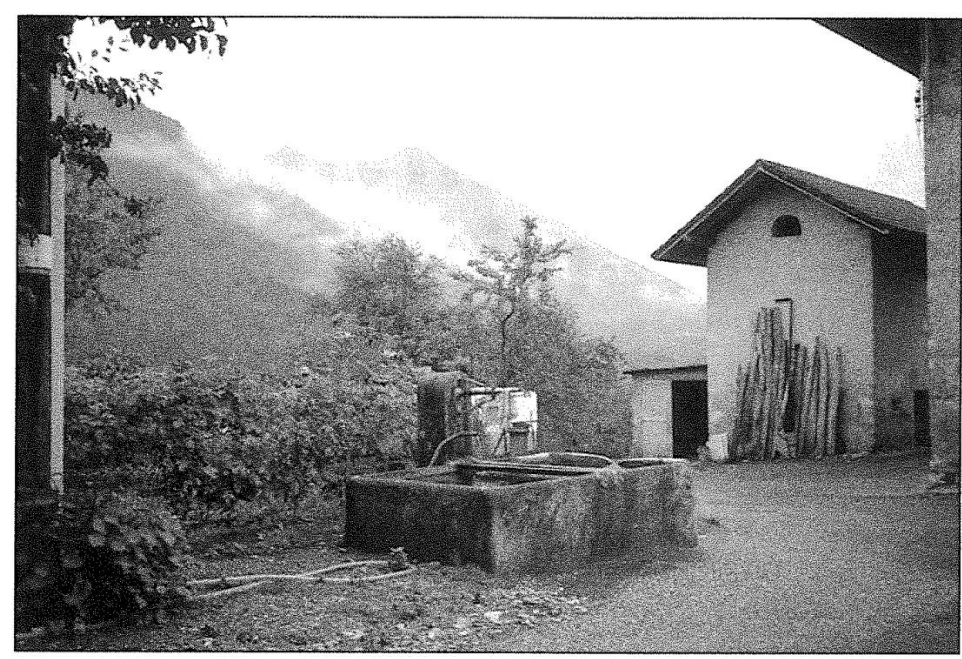
consommateur croit acheter, c'est sa propre conception de la valeur qu'il attribue au produit $»$ (Peter Drucker, cité in AFIT, 2000: 97).

\section{Conclusion : \\ la diversité des modes de développement}

Le tourisme est devenu un enjeu important, en termes de loisirs, d'emploi, d'aménagement du territoire; on a donc voulu le rationaliser, en partant du constat que l'offre dispersée, disparate, était incapable de répondre à la modernisation nécessaire et à la demande. Il est évident que cette rationalisation, par les plans successifs d'aménagement du territoire, les diverses procédures de développement local, nationales et européennes, ont permis de multiplier et d'adapter l'offre aux attentes diverses des clientèles. Toutes les techniques et les méthodes qui sont conseillées résultent de 1'analyse de situations, de réussites et d'échecs; elles proposent d'éviter des problèmes récurrents, elles donnent des idées adaptables à son cas particulier; elles corrigent des erreurs commises par des professionnels qui ne sont pas toujours compétents. Or, il faut bien constater qu'elles ne correspondent pas forcément aux manières de faire des professionnels. Elles satisfont les investisseurs d'équipements lourds, les gestionnaires d'entreprise, petite ou grande, mais elles sont étrangères aux créateurs de petites entreprises (comme ceux dont nous avons décrit la démarche ci-dessus) et à tous les prestataires qui travaillent sur un mode artisanal pour le plaisir de partager leur passion.

Cette rationalisation souffre d'un vice rédhibitoire, celui de vouloir penser à la place de l'entrepreneur. Elle assène des vérités pour les objectifs à suivre, comme pour les méthodes à appliquer. Quel que soit

\section{Bibliographie}

AEIDL (Association Européenne pour l'Information sur le Développement Local) (1997), Méthodologie pour le développement local (document de méthode).

AFIT (Agence Française de l'Ingénierie Touristique) (2000), Carnet de route de la montagne, de l'écoute des clients à l'action marketing, Paris, décembre, 155 p.

Descamps, M. (1993), Pourquoi développerle tourisme de pays, Rapport au Ministre du Tourisme, Paris, 64 p.

Dimou, A. (1989), Les néo ruraux... 20 ans après, Cemagref, Université Pierre MendèsFrance, Mémoire de DEA, Grenoble.

Fédération des PNR (2000), Guide méthodologique pour la mise en œuvre du tourisme durable, Paris.

preneur fier de son indépendance ne peut les accepter en tant que telles, parce qu'il ressent un besoin vital d'être acteur des choix qui le concernent.

Dans la situation de décalage actuel, les professionnels ont tendance à vouloir supprimer ces organismes de développement « qui ne servent à rien »; inversement, les techniciens ont tendance à éviter des professionnels « qui ne comprennent rien ». Pourtant le développement est toujours et de plus en plus une articulation entre des acteurs locaux et des acteurs extérieurs, entre des professionnels et des institutions. Les tensions sont permanentes et peuvent être des forces de progrès. La rencontre entre techniciens et professionnels suppose de reconnaître la diversité des acteurs et des modes de développement, au-delà des variantes d'un même modèle, et de redonner les responsabilités du changement social à l'ensemble des acteurs concernés. La notion de tourisme durable peut y contribuer puisqu'il recommande d' « impliquer les habitants des territoires dans les processus de décision » (principe $\mathrm{n}^{\circ} 5$ de la Charte européenne de tourisme durable).

Jacques Perret est chercheur au Cemagref (Centre national du machinisme agricole, du génie rural, des eaux et des forêts), Institut public de recherche à Grenoble.
Gramond, Florence, Joël Morette, et Jean-Pierre Portefait (1998), L'agritourisme, AFIT, Paris, $85 \mathrm{p}$.

Marcelpoil, Emmanuelle (1997), L'organisation économique du sillon alpin. Contribution à l'analyse des territoires, Thèse de Doctorat, Université Pierre Mendès-France, Grenoble, 302 p.

Marcelpoil, E., et J. Perret (1999), « Le poids conceptuel des districts industriels dans la de F. Gerbaux, Utopie pour le territoire: cohérence ou complexité ? Éditions de l'Aube. La Tour d'Aigues, p. 15-33.

Pecqueur, M. (1989), Le développement local, Syros, $149 \mathrm{p}$.

Perrot, J. (1986), « Il était une fois le développement », Berthoud, Bruyère-Rieder, Latouche, Perrot, Rist, Sabelli, Le forum du développement, Éditions d'en bas, Lausanne.

Rist, Gilbert (1996), Le développement. Histoire d'une croyance occidentale, FNSP, Références Inédites, septembre, 426 p.

Teyssandier, J.-P., et J. Perret (2000), Piloter le tourisme durable dans les territoires et les entreprises, AFIT, Paris, novembre, $78 \mathrm{p}$.

Viard, Jean (1998), Réinventer les vacances. La nouvelle galaxie du tourisme, Groupe de prospective présidé par J. Viard, Commissariat Général du Plan, Secrétariat d'État au Tourisme, La Documentation française, Paris, 333 p. construction des territoires », Sous la direction 